\title{
Analysis and Characterization of Isopropyl Carbanilate Herbicide and Its Photoproducts
}

\author{
Mohammed Fehmi Zaater \\ Department of Chemical Sciences, Jordan University of Science and Technology, Irbid, Jordan \\ E-mail:mzaater@just.edu.jo \\ Received January 17, 2011; revised May 20, 2011; accepted June 19, 2011
}

\begin{abstract}
The phototransformation of the herbicide Isopropyl carbanilate (IPC) has been investigated under UV light. Irradiation of the herbicide at room temperature in aqueous and organic solvents such as hexane and methanol afforded new photo-products formed as a consequence of various processes including photo-Fries rearrangement, ring solvolysis, hydrolysis of the amide/carbamoyl and ester bonds, ring coupling and polymerization. The percentage remaining of the herbicide as a function of time was followed periodically starting from zero time up to three hours. Analyses were performed by GC-FID equipped with a semipolar glass column operated at $170^{\circ} \mathrm{C}$. The rate of photo disappearance of IPC under controlled lab condition followed 1 st order kinetics and found to be solvent dependent in the manner of non polar $>$ polar solvents. The photo-products were successfully separated by GC and preparative TLC (Silica gel F-254) and were identified using either GC-MS and/or MS. Identifications were assigned on the bases of molecular ions, mass fragmentation pattern and whenever possible by comparison with the mass spectra of literature analogues.
\end{abstract}

Keywords: Phototransformation, Isopropyl carbanilate, Isopropyl- $N$-Phenylcarbamate, Propham, IPC and GC-MS

\section{Introduction}

Isopropyl carbanilate or Isopropyl $N$-phenylcarbamate, also known as propham, Turberite ${ }^{\circledR}$ or IPC, is a xenobiotic urethane derivative with $\mathrm{Kow}=2.6(\mathrm{pH} 7$ and $\left.20^{\circ} \mathrm{C}\right), \log \mathrm{P}=2.58$ and $\mathrm{LD}_{50} \geq 5000 \mathrm{mg} / \mathrm{kg}$. Propham has the formula of $\mathrm{C}_{10} \mathrm{H}_{12} \mathrm{NO}_{2}$ shown in Figure 1.

IPC has been widely used as a soil acting herbicide with selectivity towards weeds and grasses in broadleaved crop vegetables $[1,2]$. Once IPC finds its way into the environment, it undergoes biological, chemical and photochemical transformation [3], producing eventually the same or similar degradation products, indicating difficulties in judging the cause for a specific transformation.

Regarding biodegradation of the herbicide, it has been

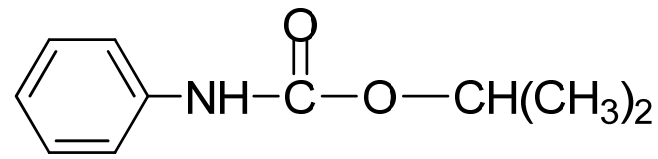

Figure 1. Chemical structure of Isopropyl- $N$-phenylcarbamate, (IPC). reported that hydrolysis to the respective aniline and isopropanol in a reverse manner to its synthesis constitutes the major metabolic pathway especially in tolerant plants and soil [4-10]. Molecular modification into more polar derivatives through hydroxylation of the aromatic and side alky moieties with subsequent conjugation to endogenous matter constitutes the principal transformation route of IPC in animals [10-12]. Additionally, a minor route for propham metabolism in animals include hydrolysis into aniline with subsequent acylation, hydroxylation and finally conjugation to qlucuronide or sulfate [13], $N$-hydroxylation and ring methoxylation of the intact IPC were also reported to occur in animals [13-15].

Previous studies pertaining to the photolysis of IPC revealed that it was highly resistant and photolysed very slowly even at short UV radiation $[16,17]$. Other studies in this context showed that IPC photolysed very slowly in a reverse manner to its synthesis giving aniline, phenylisocyanate, isopropanol and diphenylurea, all in accordance to its thermal degradation or pyrolysis products $[18,19]$. Investigations in relation to IPC persistence in the environment indicated a half-life of 254 days with 
unidentified metabolites in water under simulated summer sunshine [16]. However, in presence of $\mathrm{TiO}_{2} / \mathrm{H}_{2} \mathrm{O}_{2}$ and UV, the half-life of IPC was shortened to few hours $[20,21]$, giving a variety of degradation products including ortho- and para-hydroxy analogues, benzoquenone, isopropyl aminobenzoate and aminophenol derivatives [22-27]. In view of these conflicting reports and inadequate data on propham photo-product, herein we report the IPC photodegradation in various media with its respective photoproducts; however, such data are not fully available and the demand for it is important both to chemists and environmentalists.

\section{Experimental Studies}

\subsection{Material and Equipments}

Isopropyl- $\mathrm{N}$-penylcarbamate technical grade of purity $>$ 99\% from Sigma, solvents and reagents were of analytical grade or Analar and used as such, water used was deionised. Thin layer chromatography plates of silica gel with fluorescent indicator $\mathrm{GF}_{254}(20 \times 20 \mathrm{~cm}, 2 \mathrm{~mm}$ thickness) were used. The plates were developed with hexane-toluene- acetone $(7: 2: 1, \mathrm{v} / \mathrm{v} / \mathrm{v})$. Irradiation in solution was performed with UV light from Hanova mercury lamp (125 W) with two band-passes at 280/254 $\mathrm{nm}$ and equipped with internal water cooled quartz immersing jacket. The photolysed solutions were open to the atmosphere.

Analysis of the remaining IPC was made by GC-FID (Pye Unicam) fitted with semipolar packed OV-17 column $\left(1.8 \mathrm{~m}, 4 \mathrm{~mm}\right.$ i.d) operated at $170^{\circ} \mathrm{C}$. Mass spectra for identification were conducted on GC-MS (Hewlett Packard/5890) and MS. UV spectra were performed on UV-VIS spectrophotometer (Perkin Elmer).

\subsection{UV Spectra}

In an attempt to evaluate photolabilaty of IPC and determine its characteristics absorption maximum, a preliminary investigation on UV spectra of IPC in methanol and hexane were taken.

\subsubsection{Photoirradiation in Aqueous Solution}

One litre solution of $200 \mathrm{mg} / \mathrm{L}$ in water was introduced into a UV reactor system and exposed to UV at $254 \mathrm{~nm}$ for 3 hours at room temperature. The reactor was shielded with aluminium foil for protection. Aliquots of $25 \mathrm{~mL}$ were taken periodically every $30 \mathrm{~min}$ starting from zero time, followed by extraction with methylene chloride. The combined organic extract was washed with deionized water, dried over anhydrous $\mathrm{Na}_{2} \mathrm{SO}_{4}$, filtered and finally evaporated under reduced pressure using rotary evaporator. The remaining brown red viscous residue was reconstituted in a small amount of methanol and completed to $5 \mathrm{~mL}$ volume. The collected samples were analysed for the disappearance of IPC using GC-FID which was checked periodically for linearity using standards of the herbicide in methlyne chloride. Portions of the collected samples were also subjected for qualitative analysis by GC-MS. To examine the effect of acetone as a co-solvent and photosensitizer, the above experiment was repeated in presence of $3 \%$ acetone. The change of propham amount with time of irradiation is shown in Figure 2.

\subsubsection{Photoirradiation in Organic Solvents}

One litre solution of $200 \mathrm{mg} / \mathrm{L}$ IPC in hexane, methanol was similarly irradiated at room temperature for 3 hours. Aliquots of $5 \mathrm{~mL}$ were withdrawn periodically and analysed for disappearance of the herbicide. After the termination period the remaining solution was evaporated to dryness. Eventually the red viscous residue was redissolved in methanol and qualitatively analysed using GC-MS instrumentation.

Propham in methanol was similarly irradiated and analysed. Controlled blank experiments were reserved in a dark room.

\subsubsection{Separation and Identification of Photoproducts} The photolysate from different solvents used were carefully chromatogrammed on preparative TLC plates with fluorescent indicator. Plates were developed in a mixture of hexane-toulene-acetone $(7: 2: 1, \mathrm{v} / \mathrm{v} / \mathrm{v})$ respectively. Following development, plates were visualised under UV light, the localised bands were scrapped, taken in methanol and finally analysed by MS spectrometer.

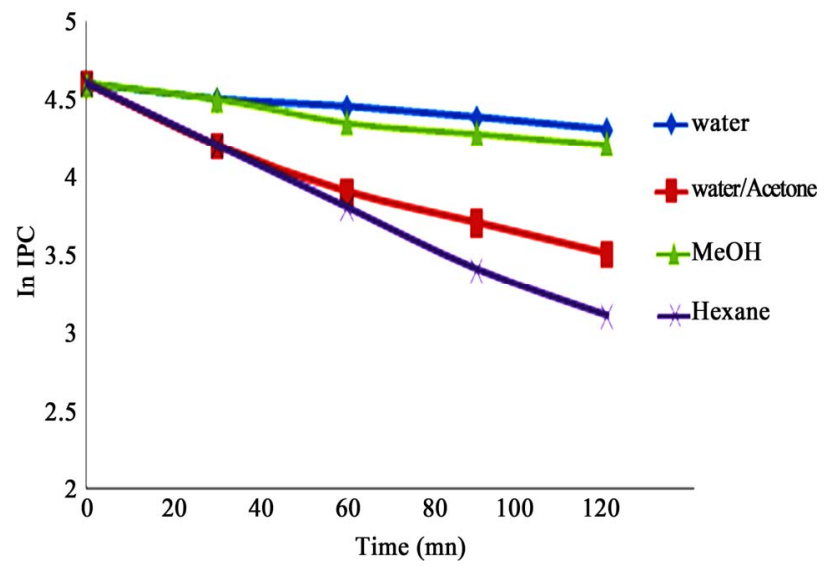

Figure 1. Photodegradation profile of In IPC (y) with time value $(x)$ in various media with regression equations for $-\mathrm{y}=0.002 \mathrm{x}+4.95, \mathrm{r}^{2}=0.990,-\mathrm{y}=0.003 \mathrm{x}+5.88$, $\mathbf{r}^{2}=0.977,-y=0.009 x+4.52, r^{2}=0.974, \longrightarrow y=$ $0.012 x+4.58, r^{2}=0.997$. 
Identifications were made on the basis of parent molecular ions $\left(\mathrm{M}^{+}\right)$, mass fragmentation pattern and whenever possible by comparison with mass spectra of literature analogues.

\section{Results and Discussion}

From the spectral data of propham in hexane and methanol it was expected that the herbicide was not a good candidate for direct photolysis. As it showed negligible absorbance above solar cut off wavelength and down to the maximum output of UV mercury lamp used. This is in accordance to the fact that only absorbed radiation is effective in producing chemical changes (Grothuss-Drapper Law). However, IPC in hexane and methanol showed two intense maxima at $\lambda\left(\varepsilon \times 10^{6}\right) 203(18.0), 233(16.5)$ and 205(14.2), 236(16.4), respectively and were assigned to $\pi-\pi^{*}$ transition of the carbonyl bond.

The undertaken preliminary lab investigation in this context suggested that IPC is a photo-susceptible compound as its solution in aqueous and organic solvents became increasingly yellow and turbid with some adhered to the wall of the photoreaction vessel, in contrast to its intact solution.

Initially the use of aqueous solvents was used because water is the most universal polar solvent available and might come into contact with the applied herbicides in the environment. The addition of acetone to aqueous solution of IPC was chosen to act as a co-solvent and a triplet photo-sensitizer that mimics the sensitising effect of humic matter in natural water [28,29]. However, the use of organic solvents was conducted to enhance the solubility of IPC and facilitate its direct injection into the chromatographic system.

The mode of degradation was carried out by monitoring its remaining concentration as a function of time compared to its initial concentration. Figure 2 demonstrates the photolysis of IPC in aqueous and organic solvents at a concentration of $200 \mathrm{mg} / \mathrm{L}$. From the representative graph it is clear that the disappearance of IPC follows $1^{\text {st }}$ order kinetic in agreement to what has been reported for most herbicides [30,31]. The photolysis rate of IPC in different solvents demonstrated similar behaviour with an order of hexane $>$ aqueous acetone $>$ methanol $>$ water. This trend is due to differences in the routes followed for transformation [32]. Homolytic cleavage with subsequent hydrogen or solvent abstraction was a general and dominant mechanism in organic solvents [32]. The slower rate in polar solvents as compared to that in hexane could be assigned to the association of the herbicide with protic solvent via hydrogen bond in agreement with literature report [22], or due to stabilization of the excited state of its bi-radical cage [ArNH
ĊOOR] [19,33,34]. The higher rate of disappearance in hexane is attributed to its hydrogen radical donating ability as compared to that of methanol and water. The GCMS and mass spectral analysis of the photolysates together with the separated photo-products revealed the formation of various compounds. The major photo-products in hexane were two isomers of IPC with molecular ions, $m / z^{+}, 197$ with different $\mathrm{R}_{\mathrm{f}}$ values and were assigned as isopropyl ortho- and para-aminobenzoate, additionally two solvolysis isomers with molecular ion $m / z^{+}, 263$ were identified as hexylpropham.

Those products were expected to proceed via homlytic cleavage of the carbamoyl bond giving either a bi-radical cage [ArN H ĊOOR] or normal free radicals ArN H \& COOR [33,34]. In both cases the radicals were able to abstract hydrogen atom and undergo concerted photoFries intramolecular rearrangements to ortho or para positions or else the radical may delocalise over the entire ring and abstract solvent molecules.

The photoirradiation of IPC in methanol provided variety of products including two isomers of ortho- and para-methoxy IPC with $\mathrm{m} / \mathrm{z}^{+}, 209$, two coupled dimers with $m / z^{+}, 356$ in contrast to the situation in hexane, hydroxyaminophenol $\mathrm{m} / \mathrm{z}^{+}, 123$, aminoqinone derivative with $\mathrm{m} / \mathrm{z}^{+}, 193$ and isopropyl aminobenzoate isomers, $m / z^{+}, 179$.

Eventually, the photolysis of IPC in aqueous media afforded two isomers of ortho- and para- hydroxy IPC, $m / z^{+}, 195$ with different $\mathrm{R}_{\mathrm{f}}$ and $\mathrm{t}_{\mathrm{r}}$ values, two coupled derivatives with $\mathrm{m} / \mathrm{z}^{+}, 356$, two isomers of IPC $\mathrm{m} / \mathrm{z}^{+}, 179$ and benzazolone, $m / z^{+}, 135$, together with an unidentified polymeric matter. The formation of benzazolone derivative is a result from internal thermal cyclisation of the respective hydroxy-IPC [16].

In this context it is worth noting that the demonstrated modes of IPC phototransformation i.e. solvolysis, photoFries intramolecular rearrangement and dimerization were not reported in any previous study. However, these have similarities with other photolysed herbicides [21, 22,33-35] or some have been reported to occur for IPC in animals [13-15]. Additionally phenylisocyanate, isopropanol, di-isopropyl ether and amino phenols were also identified from the MS spectra and could be attributed to the hydrolysis of the carbamoyl and ester bond as previously reported [18].

\section{Conclusions}

The result of this investigation revealed the photolabilaty of IPC under the influence of short UV radiation in polar and non polar media. The rate and route of IPC photodegradation were affected by the nature and polarity of the photolysed media. The principal routes of IPC disap- 
pearance were solvolysis, hydrloysis, photo-Fries concerted rearrangement and dimarization. These finding indicate the photolabilaty of IPC under UV exposure and provides valuable information both to chemists and environmentalists.

\section{References}

[1] K. A. Hassall, "The Biochemistry of Pesticides," MacMillan Press Ltd., London, 1990, pp. 313-318.

[2] I. R. Hill and S. J. L. Wright, "Pesticide Microbiology," Academic Press, London, 1978, pp. 79-136.

[3] G. G. Still and E. R. Mansager, "Aryl-Hydroxylation of Isopropyl 3-Chlorocarbanilate by Soybean Plants," Phytochemistry, Vol. 11, No. 2, 1972, pp. 515-520. doi:10.1016/0031-9422(72)80006-2

[4] H. D. Puurow, M. Canle, J. A. S. Palla and S. Steenken, "Reactions and Pathway Mechanism of Photodegradation of Pesticides," Journal of Photochemistry and Photobiology, Vol. 67, No. 2, 2002, pp. 71-108.

[5] C. Tomlin, "Pesticide Manual," 10th Edition, British Crop Protection Council, Blackwell Science Publishing, Cambridge, 1994.

[6] Farm Chemicals Handbook, Mesiter Publishing Campany, Vol. 86, 2000.

[7] G. G. Still and R. A. Herrett, "Methylcarbamate, Carbanilate and Acylanilide," In: P. C. Kearney and D. D. Kaufman, Eds., Herbicides, Chemistry, Degradation and Mode of Action, Marcel Dekker Inc., New York, 1976.

[8] C. M. Menzie, "Metabolism of Pesticides," Updated II, Special Science Report, Wildlife No. 212, U.S. Department of Interior, Washington. D. C., 1978.

[9] I.-S. You and R. Bartha, "Metabolism of 3,4-Dichloroaniline by Pseudomonas Putida," Journal of Agricultural and Food Chemistry, Vol. 30, No. 2, 1982, pp. 274-277. doi:10.1021/if00110a014

[10] J. L. Marty, T. Khafif, D. Vega and J. Bastida, "Degradation of Phenylcarbamates by Pseudomonas Alcaligenes isolated from Soil," Soil Biology and Biochemistry, Vol. 18 , No. 6, 1986, pp. 649-653. doi:10.1016/0038-0717(86)90089-1

[11] G. G. Still and E. R. Mansager, "Soybean Shoot Metabolism of Isopropyl 3-Chlorocarbanilate: Ortho and Para Aryl Hydroxylation," Pesticide Biochemistry and Physiology, Vol. 3, No. 1, 1973, pp. 87-95. doi:10.1016/0048-3575(73)90013-8

[12] D. Cole, "Oxidation of Xenobiotics in Plants," In: D. H. Huston and T. R. Roberts, Eds., Progress in Pesticide Biochemistry and Toxicology, John Wiley and Sons, New York, Vol. 3, 1983; pp. 139-1446.

[13] G. D. Paulson, A. M. Jacobsen, R. G. Zaylskie and V. J. Feil, "Isolation and Identification of Propham Metabolites from the Rat and Goat," Journal of Agricultural and Food Chemistry, Vol. 21, No. 5, 1973, pp. 804-810. doi:10.1021/jf60189a008
[14] C. C. Irving, "Enzymatic N-Hydroxylation of the Carcinogen 2-Acetylaminofluorne and the Metabolism of N-Hydroxy- 2-Acetylamino-Fluorene-4-C ${ }^{14}$ in Vitro," The Journal of Biological Chemistry, Vol. 239, 1964, p. 1589.

[15] D. L. Heikes, "Mass Spectral Identification of a Metabolite of Chlorpropham in Potatoes," Journal of Agricultural and Food Chemistry, Vol. 33, No. 2, 1985, pp. 246-249. doi:10.1021/jf00062a023

[16] N. L. Wolfe, R. G. Zepp, D. F. Parris and G. L. Baughman, "Carbaryl, Propham and Chlorpropham: A Comparison of the Rates of Hydrolysis and Photolysis with the Rate of Biolysis," Water Research, Vol. 12, No. 8, 1978, pp. 565-571. doi:10.1016/0043-1354(78)90134-3

[17] L. C. Mitchell, "The Effect of Ultraviolet Light (2537A) on 141 Pesticide Chemicals," Journal of the Association of Official Agricultural Chemists, Vol. 44, 1961, pp. 643-712.

[18] D. G. Crobsy, "Herbicide Photodecomposition," In: D. C. Kearney and D. D. Kaufman, Eds., Herbicides, Chemistry Degradation and Mode of Action, Marcel Dekker, Inc., New York, Vol. 2, 1976, pp. 835-890.

[19] G. W. Ware, "Review of Environment Contamination \& Toxicology," Google Books Results, 2000; pp. 113-116.

[20] D. Masilamani and R. O. Hutchins, "Photoinduced Rearrangement and Related Chemicals of Ethyl N-PhenylcarBamate," The Journal of Organic Chemistry, Vol. 41, 9761, pp. 3687-3691.

[21] W. Bahnemann, M. Muneer and M. Haque, "Titanium Photcatalysed Degradation of Selected Organic Pollutants in Aqueous Suspension," Catalyst Today, Vol. 124, No. 3-4, 2007, pp. 133-148. doi:10.1016/j.cattod.2007.03.031

[22] F. F. Guzik, "Photolysis of Isopropyl-N-3-Chloro-Carbani-late in Water," Journal of Agricultural and Food Chemistry, Vol. 26, No. 1, 1978, pp. 53-55. doi:10.1021/jf60215a026

[23] aeru@herts.ac.uk /footprint, 2008.

[24] T. Mill, "Chemical and Photochemical Oxidation," In: O. Hutzinger, Ed., The Handbook of the Environmental Chemistry, Springer-Verlag, Berlin, 1980, pp. 77-104.

[25] R. G. Zepp, "Experimental Approaches to Environmental Photochemistry," In: O. Hutzinger, Ed., The Handbook of the Environmental Chemistry, Springer-Verlag, Berlin, 1982, pp. 19-42.

[26] T. Mill, "Prediction of the Environmental Fate of Tetrachlorodibenzodioxin," In: M. A. Kamrin and P. W. Rodgers, Eds., Dioxin in the Environment, Hemisphere Publ. Corp, Washington D. C., 1985; pp. 173-193.

[27] D. R. Arnold and P. C. Wong, "The Photochemistry of Chloroaromatic Compunds," Journal of the American Chemical Society, Vol. 99, No. 10, 1977, pp. 3361-3366. doi: $10.1021 / \mathrm{ja} 00452 \mathrm{a} 029$

[28] G. Papageorigou and A. B. Corre, "Mechanism of Photo-Fries Photolysis of Carbamtes," Photochemical \& Photobiological Sciences, Vol. 4, 2005, pp. 216-220. 
[29] F. S. Tanaka, B. L. Hoffer and R. G. Wein, "Biphenyl Formation in the Photolysis of Aqueous Herbicide in Aqueous Media," Pest Management Science, Vol. 16, 2006, pp. 265-270.

[30] J. E. Herweh and C. E. Hoyle, "Photodegradation of Some Alkyl N-Phenylcarbamate," The Journal of Organic Chemistry, Vol. 45, No. 11, 1980, pp. 2195-2201. doi:10.1021/jo01299a031

[31] A. Kiss and D. Virag, "Photostability \& Photodegradation Pathway of Distinctive Pesticides," Journal of Environmental Quality, 2009, Vol. 38, No. 1, pp. 157-163. doi:10.2134/jeq2007.0504

[32] E. Brillas, E. Mur, R. Sanleda, et al., "Aniline Mineralization by AOP's: Anodic Oxidation, Photocatalysis, Electro-Fenton and Photoelectro-Fenton Processes," Applied
Catalysis B: Environmental, 1998, Vol. 16, No. 1, pp. 31-42. doi:10.1016/S0926-3373(97)00059-3

[33] E. S. Orejuela, "Determination of Propham and Chloropropham in Postharvest Potatoes by LC with Chemiluminescence Detection," Analytical Letters, Vol. 37, No. 12, 2004, pp. 2531-2543. doi:10.1081/AL-200031123

[34] M. Muneer, M. Qamar, M. Saquib and W. Bahnemann, "Heterogeneous Photocatalysed Reaction of Selected Pesticides in Aqueous Suspension of Titanium Dioxide," Chemosphere, Vol. 61, No. 4, 2005, pp. 457-468. doi:10.1081/AL-200031123

[35] A. Ozcan, Y. Sahin and M. Oturan, "Removal of Propham from Water by Electro-Fenton Technology, Kinetic and Mechanism," Chemosphere, 2008, Vol. 73, No. 5, 737 744. doi:10.1016/j.chemosphere.2008.06.027 